\title{
AUTOMATED PROTEIN IMMUNOBLOTTING BY PHOTOPATTERNING OF POLYACRYLAMIDE GEL IN TWO-DIMENSIONAL MICRODEVICE
}

\author{
Mei He and Amy E. Herr*
}

Department of Bioengineering, University of California, Berkeley, CA 94720, USA

\begin{abstract}
Here we report on automated protein immunoblotting using microfluidic technology. This technology utilizes a novel twodimensional microfluidic architecture photopatterned with polyacrylamide gel to yield polyacrylamide gel electrophoresis (PAGE), transfer, and in-situ antibody-based immuno-detection. The high-resolution polyacrylamide (PA) gel photopatterning provides the ability to mimic both a miniature slab gel and a protein-binding membrane, albeit with enhanced performance. In this paper, native prostate specific antigen (PSA) extracted from a human seminal fluid sample was used to demonstrate the direct mapping of analyte mobility to antibody-affinity based protein identification in a fully programmable manner. Our design paradigm has adaptable performance for relevance to all categories of immunoblotting, including the western, southern, northern and eastern blotting. The approach reported here forms the basis for a new approach to protein assays.
\end{abstract}

\section{INTRODUCTION}

Immunoblotting is exquisitely specific - allowing detection of a single protein species amidst a sea of confounding species [1]. The unrivaled specificity of immunoblotting arises from direct mapping of analyte mobility to the presence/absence of an antibody-binding interaction [2]. Immunoblotting comprises a suite of powerful protein measurement techniques used in virtually every life sciences laboratory in the world - paradoxically, the methodologies are fraught with errors, require trained operators, and demand up to two days (48 hrs) to complete [3]. Recently, automated immunoblotting has been reported using a capillary format $[4,5]$. Capillary isoelectric focusing (IEF) was used to separate proteins, including protein isoforms [6]. However, in capillary systems, integration of multiple assays is difficult without external fixturing to multiple reservoirs and interfaces. In contrast, microsystems provide a format amenable to integration of multiple functions in a single microfluidic system, which substantially benefits automation of multi-stage assays by obviating cumbersome interfacing at each channel junction [7]. The ability to fabricate interconnecting channels with zero dead volume further contributes to high performance and low sample loss. We build on additional compelling advantages of microfluidic systems that include: reduced manual intervention, nominal sample consumption, rapid results, improved assay precision, and digital
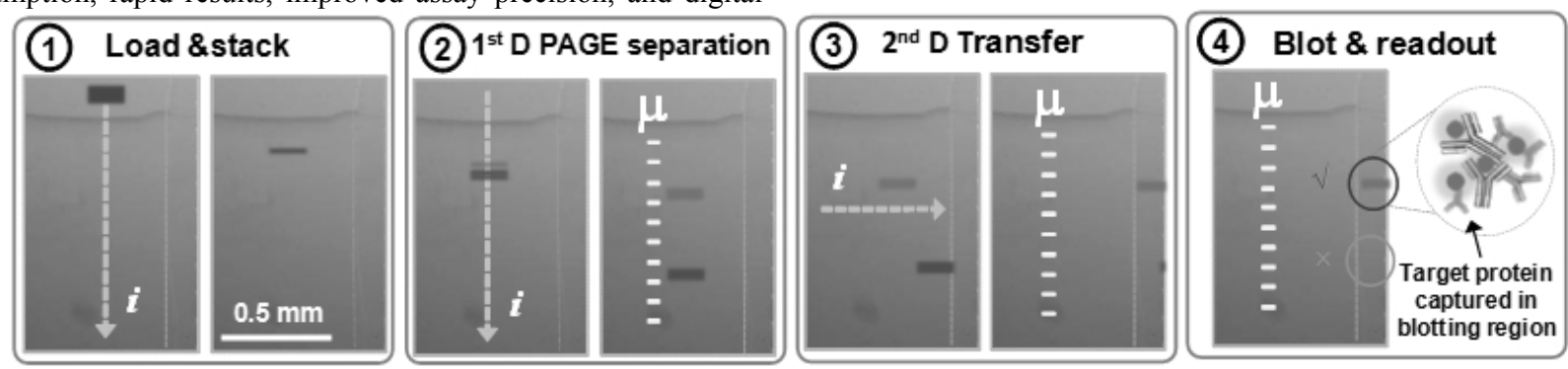

data collection essential for archiving and comparison [8, 9], which are exceptionally well-suited formats for multi-step analyses [10, $11]$.

To surmount the noted limitations of macroscale slab-gel immunoblotting our group reports here on the first known attempt to automate immunoblotting using microfluidic technology. In a significant improvement on our published work [12], the technology introduced utilizes a well-suited two-dimensional microfluidic architecture to yield protein separations in $<30 \mathrm{~s}$ followed by quantitative antibody-based protein detection, in one monolithic sub-millimeter polyacrylamide gel structure. In this report, we discussed design, fabrication, and validation of the approach that harnesses two-dimensional high-resolution polyacrylamide (PA) gel photopatterning in microfluidic device to provide both a miniature slab gel and a protein-binding membrane. To our knowledge, limited effort has been made to streamline the multiple steps needed to obtain mobility and binding-based identity information in one continuous assay [13]. Our design paradigm may have sweeping performance ramifications for all categories of immunoblotting, as well as for proteomic assays more broadly. The work detailed here forms the basis for a new approach to protein assays relevant to questions spanning basic science to clinical assays.

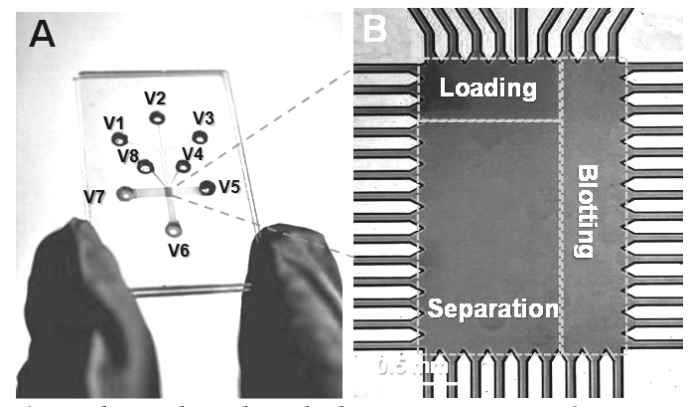

Figure 1. Polyacrylamide gel photopatterning in $2 D$ microfluidic geometry enables fully-integrated protein immunoblotting. Brightfield images of: (A) glass device housing patterned PA gels and liquid reservoirs (indicated by V1,V2,V3,V4,V5,V6,V7 and V8) for fluid access \& electrical control. (B) magnified chamber with photopatterned gels for sample loading, PAGE separation and transfer to antibody functionalized blotting region (right side).

Figure 2. The automated multi-stage assay protocol (1)-(4) consists of sample loading and stacking, vertical dimension separation, horizontal dimension transfer, and immuno-detection. " $\mu$ " indicates mobility; " $i$ " indicates direction of electrical current flow. 


\section{Experiment}

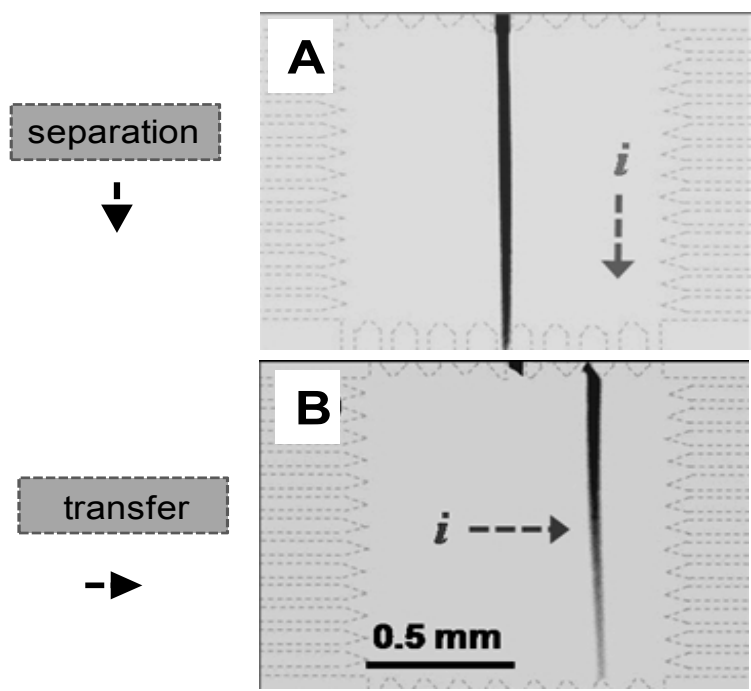

Figure 3. Dispersionless protein injection and transfer in chamber. (A) Vertical electric field for separation; (B) horizontal electric field for sample transfer to blotting region. Electrophoretic transfer visualized by loading and manipulating $0.1 \mathrm{M}$ free dye.

\section{EXPERIMENTAL}

\section{Gel Photopatterning}

High resolution regional photopatterning of PA gel precursor solutions allows fabrication of spatially-varying pore-size and chemical functionality in microfluidic devices with minimal integration dead volume. In this paper, the gel regions were sequentially photopatterned using a three-step process. Patterning was conducted in glass microfluidic chambers. The photopatterning approach provides a crucial tool for integrating the immunoblotting functionality (Figure 1A). The lithography was accomplished via a UV objective (UPLANS-APO $4 \times$, Olympus) in combination with a transparency film mask and epi-fluorescence microscope system (IX-70, Olympus, Melville, NY). Using this photolithography approach, the blotting gel was fabricated by exposing a region filled with a $6 \% \mathrm{~T}$ precursor solution (including streptavidin-acrylamide) to UV light for $8 \mathrm{~min}$. Here, the neutral density filter was used to achieve a UV intensity at $\sim 13 \mathrm{~mW} / \mathrm{cm}^{2}$. The notation $\% \mathrm{~T}$ and $\% \mathrm{C}$ indicate the percentage of total acrylamide and cross-linker, respectively. Here covalently bonded streptavidin in the gel matrix was used to immobilize biotinylated antibodies for immunoblotting and detailed in reference [14]. This functional immunoblotting region is indicated in Figure 1B. The unpolymerized region was replaced by introducing the second gel precursor solution through vacuum. In the second step, mask alignment to the chip was performed using a manual adjust $x-y$ translation stage on the microscope (Olympus, Melville, NY) to subsequently photopattern the $6 \% \mathrm{~T}$ separation gel $(5 \mathrm{~min})$. The PAGE separation gel had a composition and structure similar to the blotting matrix albeit with no immobilized antibodies present. Finally, in the third step, a larger pore-size loading gel was formed using 3\%T acrylamide solution and an 8-min flood exposure of the chip to a filtered mercury lamp (300-380 nm, $10 \mathrm{~mW} / \mathrm{cm} 2$, UVP B100-AP, Upland, CA) with cooling fan. Here the photopolymerization times were determined empirically based on the UV intensity, composition of acrylamide precursor solution, and desired pore-size to achieve optimal gel performance for the desired functions.

\section{Electric Field Simulation and Control}

To control sample dispersion common in chamber-like geometries, an array of field-control channels was included in the chip chamber design. Simulation of the load and transfer electric fields was performed using COMSOL Multiphysics (Version 3.5a, COMSOL AB). The simulation was conducted under twodimensional conductive media DC conditions. Modeling of the electrostatics within a buffer filled microfluidic channel network under variable voltages provided guidance on the optimized voltage program for experimental operation.

After sample addition to the chip, assay operation was programmable and controlled via a power supply equipped with platinum electrodes (Caliper Life Sciences). The sample (V1), sample waste (V3), buffer (V2, V4, V7, V8), and buffer waste (V5, V6) reservoirs are indicated in Figure 1A. To assure minimal cross-contamination between samples and reproducible runs, all channels were electrophoretically flushed with buffer every other run.

\section{RESULTS}

We utilize our multi-stage photopatterning approach to locate several PA gel regions (sample loading, PAGE separation and immunoblotting) in one microfluidic chamber. The chip (geometry and gel) design yields both a miniature slab gel and a proteinbinding membrane, as shown in Figure 2. After vertical PAGE separation, the resolved protein species are transferred laterally across the $2 \mathrm{D}$ chamber and target proteins are in-situ identified by antibodies immobilized in blotting region. Importantly, after sample loading and PAGE separation, our chip design enables blotting of all protein bands against the blotting region in a single electrophoretic transfer step that is fully programmable, as illustrated in Figure 2 (1)-(4).

The lateral transfer step links the vertical separation position to horizontal affinity-based binding information, thus providing a 1:1 mapping of mobility to antibody interaction. Retaining information about protein mobility, as well as binding interaction (or lack thereof) for each resolved species in a sample is critical to developing and completing a successful immunoblot. Therefore, we investigated means to achieve a uniform and efficient transfer of resolved species from the separation dimension to the blotting region of the chamber. As described in the experimental section, we employed numerical simulations to determine the applied electrical potentials to achieve minimal dispersion in sample loading, separation, transfer and blotting stagers. The experimental data showed a well-controlled transfer process in both the vertical and horizontal dimensions (Figure 3). In contrast to the injection with no electric field shaping, appropriate voltage control ensured that the injected sample plug preserved its original shape with little distortion after entering into the wider separation chamber. A uniform electric field distribution within the chamber for each step provided the basis for precisely manipulating the sample during separation and transfer.

To optimize the first step (PAGE), we use a discontinuous gel (large-to-small pore-size) near the sample injector to minimize injection dispersion for improved separation resolution (SR, where $\mathrm{SR}>1$ is desired). Here the discontinuity was created by $3 \% \mathrm{~T}$ loading gel adjacent to $6 \% \mathrm{~T}$ separation gel, and used to differentially retard the migration of proteins based on their size. Further, the separation performance benefits from the "stacking" 
effect and decreased the protein band dispersion. As compared in Figure 4, with the gel discontinuity, PAGE separation achieves an $S R=1.5$ for the two highest mobility peaks in Figure 4A. The separation was completed in 30 seconds in just a fraction of the separation axis length. While without the stacking gel discontinuity (i.e., a uniform $6 \% \mathrm{~T}$ gel), a low separation resolution of $S R=0.4$ was achieved for the same two species (Figure 4B).
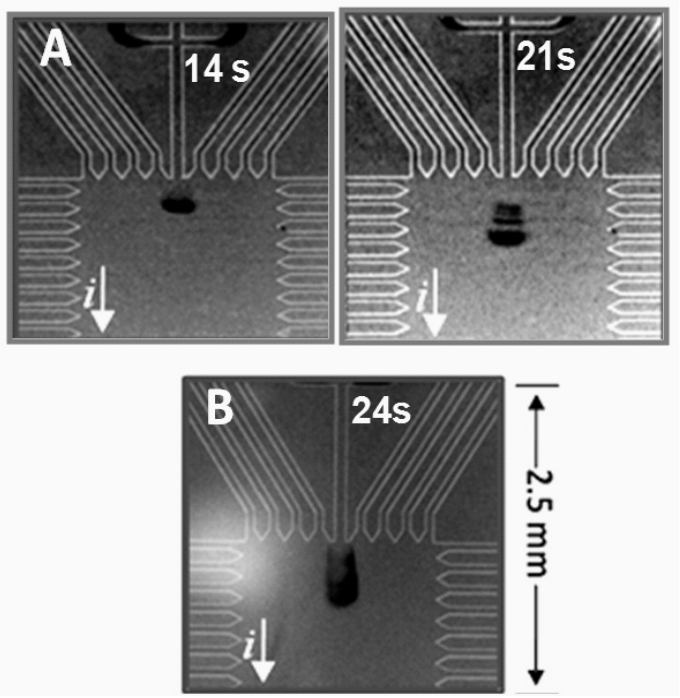

Figure 4. Discontinuous stacking gel improves separation resolution. (A) Large-to-small pore-size gel discontinuity at PAGE start yields ultra-efficient size-based PSA sample stacking and separation, compared with (B) no stacking gel. (inverted grayscale)

To demonstrate this automated immunoblotting format, we assay native free prostate specific antigen (fPSA) extracted from human seminal fluid (Figure 5). Free PSA is a tumour marker currently used for serum diagnosis of prostate cancer [15]. Much recent interest centers on improving prognostic performance through analysis other forms of PSA, including the glycosylation, and alternate diagnostic fluids (non-serum) [16].

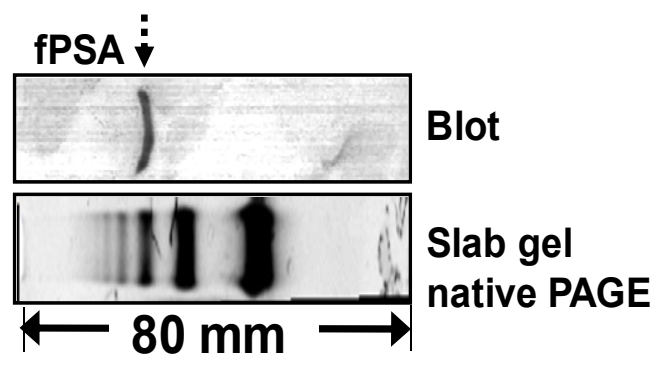

Figure 5. Gold-standard native immunoblotting analysis of the human seminar fluid extract shows multiple proteins resolved in the native PAGE step, with a single band (marked fPSA) binding to the fPSA antibody in the blotting step.

Using our microfluidic immunoblot, the PSA sample was analyzed using full automation of the protocol (i.e., no manual intervention), as shown in Figure 6. The sample was separated by native PAGE within $36 \mathrm{~s}$ with an $S R$ of 1.59. After PAGE, the onchip transfer step required $30-60 \mathrm{~s}$. The on-chip approach is significantly faster compared with $2 \mathrm{~h}$ in slab-gel systems. In the blotting stage, species exhibiting an affinity for the blotting membrane were retained in the blotting region, while species with low-to-no affinity freely migrated through the blotting region, as desired for the three non-fPSA proteins (Figure 6). The fPSA immunoblot was completed in $65 \mathrm{~s}$, a time reduction of $10,000-$ fold compared with conventional slab-gel. We also note the importance of conserving the separation resolution of the PAGE in transfer and subsequent blotting (Figure 7). Large dispersion associated with the transfer or blotting essentially "smears" the separation information and results in loss of information regarding the sample. For conventional slab gel immunoblotting, the transfer efficiency indicates the amount of transferred sample during the transfer step from gel to membrane, which is calculated by comparing the amount of protein in the gel before transfer and the amount of protein on the membrane after transfer [17]. Here, the calculated average on-chip transfer efficiency for the PSA sample is $89.5 \%$., which is comparable to conventional slab gel transfer. The conventional native slab mini-gel blot of the same PSA sample showed consistency with on-chip data.

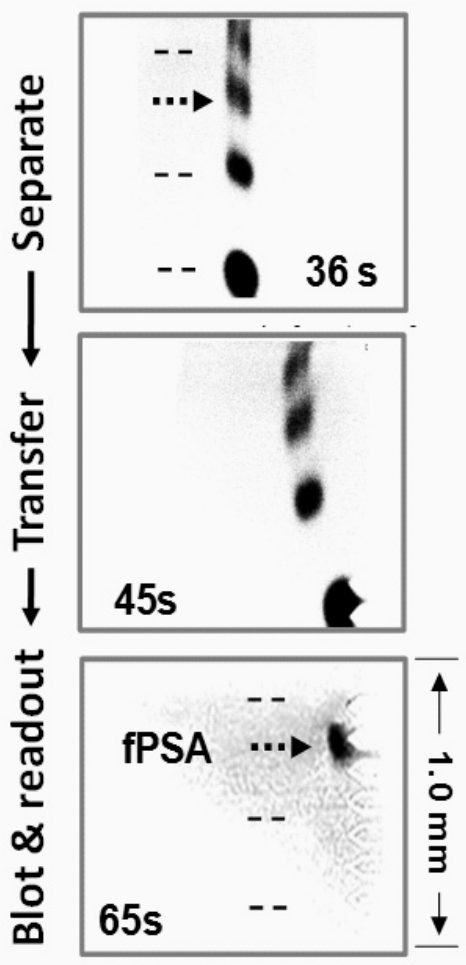

Figure 6. On-chip immunoblotting of native fPSA extracted from human seminal fluid is automated and completes in 36-seconds (compared to 1-2 days for slab-gels). CCD images show the PA gel-patterned chamber during: separation, transfer and blot. As indicated in the figure, the blotting chamber is patterned with multiple functional regions of $P A$ gel for optimized performance.

Previously, we developed two general strategies for antibody immobilization, which afford application flexibility for the native PAGE in-situ immunoblot [12]. While not demonstrated in the work presented here, the approaches are applicable to this two 
dimensional microdevice. In strategy one, streptavidin PA precursor is copolymerized with biotinylated antibodies during photopatterning step. Strategy two provides the more flexibility, which allows end-user to select the antibodies by decoration of the blotting gel containing streptavidin only through post-introducing. Additionally, it required less biotinylated blotting reagents (e.g., antibodies, aptamers, Fab fragments) in the patterning well, typically $10 \mu \mathrm{L}$ of $\sim 1 \mu \mathrm{M}$. [12]

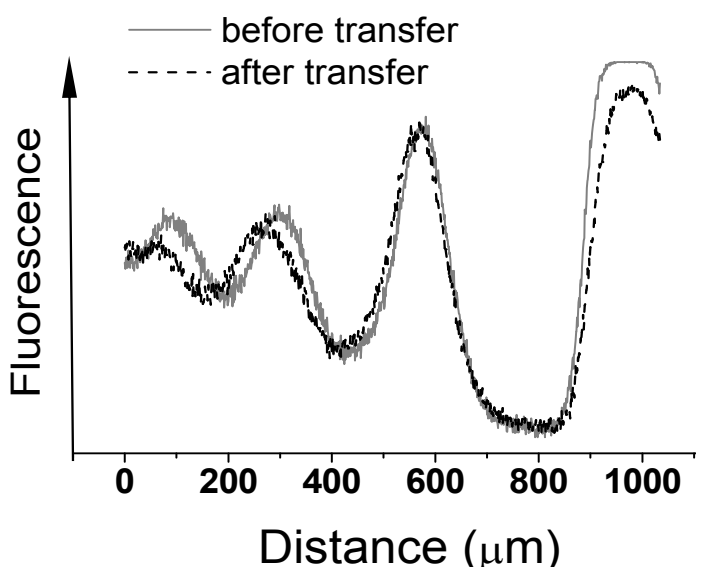

Figure 7. Dispersion associated with transfer or blotting steps can reduce the information content of the complete assay. Further, shifts in the position of protein peaks (also arising from transfer or blotting) reduce the total informational content of the assay. Through using an optimized electrophoretic transfer of species from the separation axis to the blotting gel, the approach presented here results in little transfer-associated dispersion and little peak shifting.

\section{CONCLUSION}

We introduce an effective, rapid immunoblotting assay that utilizes a single microfluidic chamber photopatterned with heterogeneous gel elements to unify PAGE protein separation, electrophoretic transfer, and antibody-based in-gel blotting. Mapping of the separation axis to the blotting region yields both electrophoretic mobility and binding information for each resolved protein. The transfer of resolved protein peaks to a blotting gel region yields no discernable sample loss and high transfer efficiency.

Initial demonstration of native PSA on-chip immunoblotting indicates that the scheme is an effective, high specific approach, suggesting a well-suited format for automated, quantitative microfluidic immunoblotting. Multiple protein peaks were detected in sample extracted from human seminal fluid, both by our new method and in comparative gold-standard PAGE slab gels. While the PAGE slab gel comparisons required up to two days to complete, the automated approach completed in $\sim 1$ minute.

Currently, optimization of the blotting gel function, enhanced applicability through integration of the immunoblot with protein sizing (sodium dodecyl sulfate PAGE), and analysis of complex protein samples relevant to both basic science and clinical questions are underway, as well as further improvements in detection sensitivity through on-chip protein enrichment. Additional design goals include reproducible quantitation (blot-to- blot reproducibility) with minimal sample consumption are also underway and can be achieved using the fine control of protein transfer demonstrated here.

We see great potential to extend the basic assay developed and presented here to panels of unique blotted proteins. The approach demonstrated here holds substantial promise as the basis for a suite of automated immunoblotting technologies, and it is applicable to a broad range of multi-stage assays, including DNA/RNA immunoblotting assays and multi-dimensional separation strategies relevant to both basic science and clinical questions.

\section{ACKNOWLEDGEMENT}

We thank Prof. D. Peehl (Stanford) for providing the PSA sample, as well as Dr. Yong Zeng and Mr. Samuel Tia for their assistance. We thank the University of California, Berkeley, the QB3/Rogers Family Foundation Award, and UC Berkeley NSF COINS for financial support.

\section{REFERENCE:}

[1] J. M. Gershoni, G. E. Palade, Anal. Biochem., 131, 1 (1983).

[2] H. Towbin, T. Staehelin, J. Gordon, Proc. Natl. Acad. Sci. USA, 76, 4350 (1979).

[3] Y. Wu, Q. Li, X. Z. Chen, Nat. Protoc., 2, 3278 (2007).

[4] N. A. Guzman, S. S. Park, D. Schaufelberger, L. Hernandez, X. Paez, P. Rada, A. J. Tomlinson, S. Naylor, J Chromatogr B, 697, 37 (1997).

[5] M. C. Peoples, T. M. Phillips, H. T. Karnes, J Pharmaceut Biomed, 48, 376 (2008).

[6] R. A. ÓNeill, A. Bhamidipati, X. Bi, A. Deb-Basu, L. Cahill, J. Ferrante, E. Gentalen, M. Glazer, J. Gossett, K. Hacker, C. Kirby, J. Knittle, R. Loder, C. Mastroieni, M. MacLaren, T. Mills, U. Nguyen, N. Parker, A. Rice, D. Roach,D. Suich, D. Voehringer, K. Voss, J. Yang, T. Yang, P.B. V. Horn, Proc, Natl. Acad. Sci. USA, 103, 16153 (2006).

[7] C. Hou, A. E. Herr, Electrophoresis, 29, 3306, (2008).

[8] M. G. Mauk, B. L. Ziober, Z. Chen, J. A. Thompson, H. H. Bau, Ann. N. Y. Acad. Sci. 1098, 467, (2007).

[9] J. West, M. Becker, S. Tombrink, A. Manz, Anal. Chem., 80, 4403, (2008).

[10]S. H. Yeung, P. Liu, N. D. Bueno, S. A. Greenspoon, R. A. Mathies, Anal. Chem., 81, 210, (2009).

[11]R. J. Meagher, A. V. Hatch, R. F. Renzi, A. K. Singh, Lab Chip, 8, 2046, (2008).

[12]M. He, A. E. Herr, Anal. Chem., 81, 8177 (2009).

[13]A. C. Fan, D. Deb-Basu, M. W. Orban, J. R. Gotlib, Y. Natkunam, R. ÓNeill, R. A. Padua, L. Xu, D. Taketa, A. E. Shirer, S. Beer, A. X. Yee, D. W. Voehringer, D. W. Felsher, Nat. Med., 15, 566 (2009).

[14]M. He, A. E. Herr, J. Am. Chem. Soc., 132, 2512 (2010).

[15]M. H. Black, C. L. Grass, J. Leinonen, U. H. Stenman, E. P. Diamandis, Clin. Chem., 45, 347 (1999).

[16]S. Prakash, P. W. Robbins, Glycobiology, 10, 173 (2000).

[17]P. Jungblut, C. Eckerskorn, F. Lottspeich, J. Klose, Electrophoresis, 11, 581, (1990).

\section{CONTACT}

*A. E. Herr, tel: +1-510-666-3396; aeh@berkeley.edu 